\title{
Next-generation Automation and Remote-access Crystallography
}

\section{Aina Cohen (representing the entire SMB team)}

Structural Molecular Biology, Stanford Synchrotron Radiation Lightsource (SSRL) and Linac Coherent Light Source (LCLS), SLAC National Accelerator Laboratory, Stanford University, Menlo Park, United States of America

\author{
acohen@SLAC.Stanford.edu
}

Structural biologists are undertaking increasingly challenging projects including the study of membrane proteins and complex multicomponent machines. Structural investigations are also transitioning beyond solving a single static structure, to the application of a series of sequential structural snapshots to provide details of the atomic positions and motions that define the relationships involved in molecular recognition, transition state stabilization, and other aspects of the biocatalytic process. The success of these experiments requires careful optimization of samples and experimental setups, often involving multiple experiments at the laboratory bench and the beamline, where automation serves as an enabling technology to efficiently deliver multiple crystals and meet stringent timing requirements.

Developments at SSRL and LCLS-MFX will be presented that tackle challenges involved in the use of very small and radiationsensitive crystals. To facilitate the handling and optimization of delicate crystals, new in situ crystallization and remote data collection schemes have been released that avoid direct manipulation of crystals, support robotic sample exchange, and allow full rotational access of the sample in a controlled humidity environment. By simplifying crystal handling and transport at near-physiological temperatures, these technologies remove barriers to enable more widespread use of serial crystallography methods for studies of metalloenzyme structure and protein dynamics. Data analysis tools that provide rapid feedback for experimental optimization during fast-paced experiments will also be described.

Keywords: structural biology, automation, serial crystallography, controlled humidity 